\title{
Neoestructuralismo y Macroeconomía para el Desarrollo ${ }^{1}$
}

\author{
Ricardo Ffrench-Davis
}

UnIVERSIDAD DE CHILE

\section{RESUMEN}

El objetivo de esta contribución es examinar los sesgos depresores y regresivos sobre el crecimiento económico y el empleo que provoca el estilo aún predominante de políticas macroeconómicas inspiradas en el Consenso de Washington o enfoque neoliberal, y delinear los rasgos y efectos centrales de una macroeconomía para el desarrollo; se enfatizan, en particular, las implicancias de la fuerte heterogeneidad estructural entre diversos tipos de agentes económicos, heterogeneidad que es un rasgo más característico de las economías en desarrollo como las de la región.

Palabras Clave: Neoestructuralismo, marcroeconomía, heterogeneidad, Desarrollo, crecimiento económico

\section{Abstract}

The objective of this contribution is to examine the depressive and regressive biases on economic growth and employment caused by the still predominant style of macroeconomic policies inspired by the Washington Consensus or neoliberal approach, and to delineate the central features and effects of a macroeconomy for the developing; In particular, the implications of strong structural heterogeneity (H.E.) among different types of economic agents are emphasized, a heterogeneity that is a more characteristic feature of developing economies like those of the region.

Keywords: Neo-structuralism, marcroeconomy, heterogeneity structural, Development, economic growth

*rffrench@fen.uchile.cl 


\section{INTRODUCCIÓN}

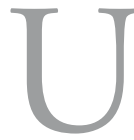

na de las responsabilidades fundamentales del Estado para el desarrollo económico y social se refiere al entorno global en que se desenvuelven los diversos productores y consumidores de bienes y servicios que operan en la economía nacional; eso es la macroeconomía. Dos rasgos básicos que debe abordar el diseño del entorno macroeconómico son que posibilite el uso pleno de los recursos productivos, con balances internos y externos sostenibles, y que ello sea efectuado de manera consistente con la formación de nuevas capacidades. Entre otros, se requiere una evolución de la demanda interna alrededor de la capacidad productiva o PIB potencial y precios macroeconómicos (en particular, el tipo de cambio) en niveles compatibles con un balance externo sostenible. Es lo que se ha llamado macroeconomía para el desarrollo, funcional para una estrategia de crecimiento con equidad.

El objetivo de esta contribución es examinar los sesgos depresores y regresivos sobre el crecimiento económico y el empleo que provoca el estilo aún predominante de políticas macroeconómicas inspiradas en el Consenso de Washington o enfoque neoliberal, y delinear los rasgos y efectos centrales de una macroeconomía para el desarrollo; se enfatizan, en particular, las implicancias de la fuerte heterogeneidad estructural (H.E.) entre diversos tipos de agentes económicos, heterogeneidad que es un rasgo más característico de las economías en desarrollo como las de la región.

Esta H.E. se manifiesta en la diversidad de productividades entre empresas de diferentes dimensiones y entre trabajadores de diversa calificación, en la diversa capacidad de acción y reacción de los agentes típicos en distintos mercados -ya sean grandes y pequeños empresarios; trabajadores de alta y de reducida calificación; inversionistas productivos generadores de PIB e inversionistas financieros buscadores de "rentas económicas" (rent seekers); inversionistas productivos y consumidores-, y en las asimetrías en las capacidades de respuesta de diferentes agentes ante la inestabilidad de la actividad económica y los macro-precios.

Las diversas asimetrías que caracterizan al comportamiento de la 
economía real, se intensifican cuanto mayor sea la inestabilidad del entorno macroeconómico. Ello reviste gran relevancia, dado que las economías de la región se caracterizan por reiteradas expansiones seguidas por situaciones recesivas (procesos de stop and go), que implican niveles promedio de la producción efectiva por debajo de la capacidad productiva del trabajo y el capital, con intensos altibajos de macro-precios como el tipo de cambio e iliquidez del mercado crediticio, y grandes fluctuaciones del balance externo. Estas diversas formas de inestabilidad exhiben una fuerte asociación con fluctuaciones de los flujos de capitales financieros y de los precios de exportaciones primarias.

La CEPAL ha planteado en La Hora de la Igualdad (CEPAL, 2010) que la combinación de heterogeneidad e inestabilidad presenta desafíos profundos al diseño de las políticas públicas: si ello no se tiene en consideración, suele fracasarse tanto en el logro del crecimiento como de la igualdad. En efecto, políticas supuestamente "neutrales" del neo-liberalismo suelen surtir efectos fuertemente negativos en ambas dimensiones: (i) regresivos, contra las PYMEs, producciones incipientes y trabajadores de menor calificación; (ii) deprimen la utilización de la capacidad productiva disponible, la formación de capital, la calidad de las exportaciones y del empleo, y la innovación. Por consiguiente, la reducción de la H.E. y de la inestabilidad de la macroeconomía real son determinantes del logro del desarrollo, el que por definición es inclusivo.

En consecuencia, las políticas macroeconómicas deben tener en consideración la heterogeneidad estructural existente para lograr nivelar la capacidad de respuesta de diversos agentes. Ello es esencial para el desarrollo, el que pasa ineludiblemente por la reducción persistente de las brechas de productividad; elevando la productividad media y reduciendo su dispersión.

Alcanzar estas metas requiere políticas articuladas y consensuadas en varios frentes, entre ellos, las políticas macroeconómicas, de desarrollo productivo y social. Bajo este enfoque, las políticas macroeconómicas pueden contribuir a transformar las estructuras productivas con un sesgo igualador de las oportunidades y a impulsar 
un dinamismo económico que acreciente la formación de capital, el empleo e innovación. Aquí nos concentramos en la dimensión macroeconómica y su influencia sobre la equidad, la productividad efectiva y la formación de capital.

Luego de esta introducción, la sección 2 presenta un breve recuento del desempeño macroeconómico de la región desde 1990. La sección 3 examina tres tipos de asimetrías que, ante la existencia de inestabilidad de la economía real y heterogeneidad estructural, resultan recesivas y depresoras del desarrollo, con impactos sobre la formación de capital, sobre la calidad de la inserción externa y sobre el nivel de empleo y su precariedad. La sección 4 concluye.

\section{El ENFOQUe DOMINANTE DESDE I 990 Y SUS EFECTOS}

Una visión dominante en estos años ha sido que la inflación y balances fiscales bajo control bastaban para asegurar la presencia de equilibrios macroeconómicos; muchos reformadores pensaban que el logro de estos objetivos se facilitaría con un tipo de cambio libre y con la apertura plena de la cuenta de capitales, lo que contribuiría a "importar" estabilidad macroeconómica al acotar eventuales acciones irresponsables de las autoridades locales; según ese enfoque, los agentes financieros, con sus votos de confianza o desconfianza respecto a las economías nacionales, ponen coto a la tendencia de las autoridades nacionales a generar desequilibrios fiscales y monetarios; a su vez, los mercados financieros, con sus flujos de fondos, determinarían niveles de liquidez, de tasas de interés y de tipos de cambio de equilibrio. Adicionalmente, la apertura de la cuenta de capitales atraería ahorro externo que se agregaría al ahorro nacional, para así financiar una mayor formación de capital.

Como ingrediente central de ello, se procuró aislar a la gestión monetaria de presiones políticas. Esto involucró tender a que la conducción de la política monetaria la efectuaran Bancos Centrales independientes y se centrara en el control de metas de inflación como objetivo dominante; con frecuencia, la apreciación cambiaria provocada por ingresos de capitales fue recibida con beneplácito por su impacto represor sobre el IPC, sin considerar su incidencia 
negativa sobre la producción de transables y la estabilidad de la economía real.

En consecuencia, en los decenios recientes, en América Latina ha solido primar un enfoque macroeconómico con una clara prioridad por la estabilidad nominal o nivel de precios. Por consiguiente, el diseño y evaluación de las políticas macroeconómicas se ha disociado de la consideración de sus efectos sobre el empleo y el crecimiento, enfocándolas en exceso en el control de la inflación. Este enfoque ha sido exitoso en el control de la inflación cuyos niveles se situaron en un dígito para la mayor parte de los países de la región hacia mediados de la década de los noventa. No obstante, no ha dado crecimiento sostenido del PIB ni de los ingresos laborales (FfrenchDavis, 2005, Introducción y 2010).

La evolución del crecimiento del PIB, durante el predominio del Consenso de Washington, lo ha hecho a un ritmo desilusionante (Williamson, 2003). Entre 1990 y 2012 un aumento del PIB de apenas 3,2\% anual, que contrasta con el 5,6\% registrado en los setenta. En efecto, la región, en su conjunto, no ha logrado convergencia, sin poder acortar distancia con el mundo desarrollado. Por ejemplo, en 2008 (el ańo previo a la llegada del contagio de la crisis global), el PIBpc de América Latina representó el mismo 27\% del ingreso medio de los países del G-7 que había alcanzado a fines de los 80s (cuadro 1); esto es, ambos grupos de naciones exhibieron un aumento similar de su ingreso por habitante en 1990-97, años de más intensa aplicación de las reformas del Consenso de Washington en la región. En los años siguientes, perdió terreno hasta 2003 bajo el influjo recesivo de la crisis asiática, y se recuperó con la llegada del shock positivo de términos del intercambio; en 2008, con una fuerte aceleración desde 2004, la región se había situado levemente sobre 27\% del PIBpc del G-7 en 2008, ${ }^{2}$ y en 2012 había avanzado al 29\%; por lo tanto, apenas 2 de los 73 puntos de brecha vigentes en 1989 fueron recortados por América Latina en un extenso período de 23 años. 
Cuadro i. Pib per Cápita de américa latina como \% De EeUu y G-7, I970-20 I 2 (en \% en ppa us\$ 2012)

\begin{tabular}{ccc}
\hline Año & América Latina/EEUU & América Latina/G-7 \\
\hline 1970 & 26,5 & 32,5 \\
1980 & 30,3 & 35,6 \\
1989 & 23,5 & 27,2 \\
1997 & 23,0 & 26,6 \\
2008 & 23,3 & 27,4 \\
2012 & 24,6 & 29,1 \\
\hline
\end{tabular}

Fuente: Los niveles de PIBpc a ppa (ppp, purchasing power parity, en inglés) se anclaron de acuerdo a las estimaciones del Banco Mundial para las 3 unidades en el año 2012, y se estimaron los niveles para los años anteriores en base a las tasas de variación real del PIBpc reportadas por el Banco Mundial entre 1970 y 2012.

Luego del contagio de la crisis global, en 2008-09, se registró una nueva recuperación vigorosa del PIB en 2010-11 (5,8 y 4,4\%, respectivamente); no obstante, otra vez, al combinar la caída por el contagio con la recuperación posterior, se mantiene un promedio similar y deficiente. A su vez, ya en el 2012 se había retornado a un $3 \%$. Ello refleja la debilidad de políticas micro (que no cubrimos en esta ocasión) y macroeconómicas.

Lo habitual ha sido que las etapas de auge -iniciadas en 199091,1995-96, 2003-04 y 2010- sean acompañadas por la generación de desequilibrios macroeconómicos en tipos de cambio, balance externo, burbujas en los precios de la bolsa y créditos de consumo, los que crecientemente se van tornando insostenibles, y que abren camino a un nuevo ajuste recesivo. ${ }^{3}$ Estos desequilibrios $-\mathrm{o}$ indicadores de vulnerabilidad-subyacen en la intensa inestabilidad que exhibe la evolución del PIB de la mayoría de las economías medianas y grandes de la región, los que por su peso le imprimen su sello a los promedios ponderados de América Latina (ver gráfico 1). Es un hecho irrefutable que, en la mayoría de la región, los cambios en la evolución del PIB no responden a cambios abruptos estructurales o en la microeconomía, sino que a variaciones cíclicas en variables macroeconómicas predominantemente asociadas al patrón de inserción financiera y comercial. 
Gráfico 1. América Latina: Volatilidad del PIB, 1977-2012 (tasas anuales de crecimiento, \%)

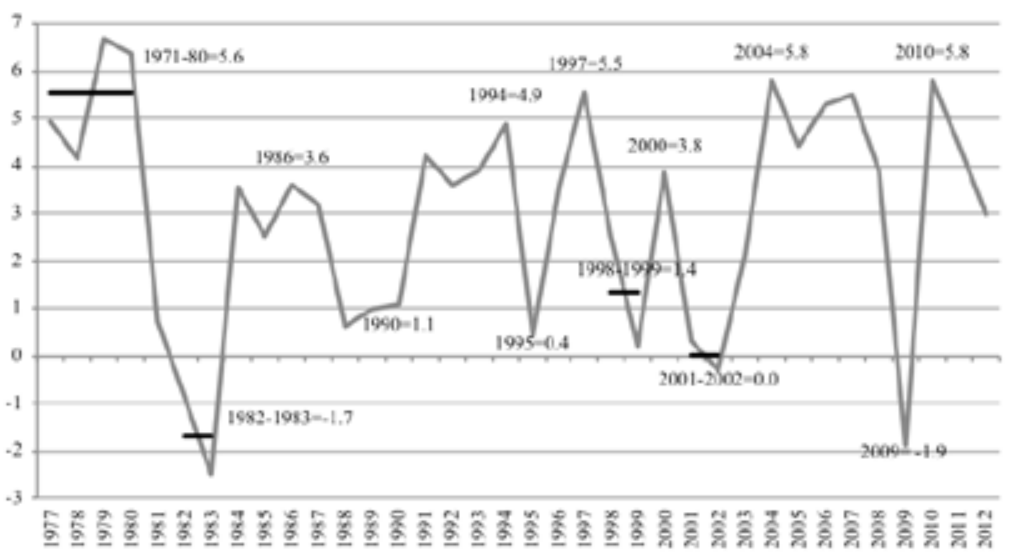

Fuente: Basado en datos de CEPAL (2013a) para 19 PALs.

Las fluctuaciones de la actividad económica estuvieron asociadas principalmente a flujos de capitales hasta 2003. El auge desde entonces, particularmente en Sudamérica, respondió a mejoras prolongadas de los términos del intercambio en vez de uso de ahorro externo; esto es, ahora se sostuvo más en fondos propios en vez de ajenos. Junto con la adopción de algunas reformas contra-cíclicas, tales como la creación de fondos soberanos estabilizadores, le ha dado cierta mayor sostenibilidad temporal. No obstante ello, la mayoría de los indicadores de vulnerabilidad macroeconómica estuvieron crecientemente presentes hasta 2008 y el proceso desestabilizador se repitió en 2010-12.

El más notorio consiste en las persistentes revaluaciones cambiarias y su impacto en las cuentas corrientes. Ellas parecen sostenibles mientras se mantengan los precios notablemente elevados de las exportaciones primarias de varios países de la región, los que le imprimen su sello a los términos del intercambio de la región; el gráfico 2 muestra la notable alza registrada por los términos del intercambio desde 2003 en comparación con los noventa. ${ }^{4}$ Sin embargo, las revaluaciones impulsadas por alzas de los términos de intercambio que suelen ser transitorias, van dejando sus huellas distorsionadoras 
en la calidad de las exportaciones y en la competitividad de las PYMES frente a importaciones cuyo volumen creció 108\% desde 2003, en contraste con el $47 \%$ del volumen de las exportaciones (gráfico 3).

\section{Gráfico 2. América Latina: relación de precios de intercambio de bienes y servicios, 1990-2012}

(indice $1990=100$ )

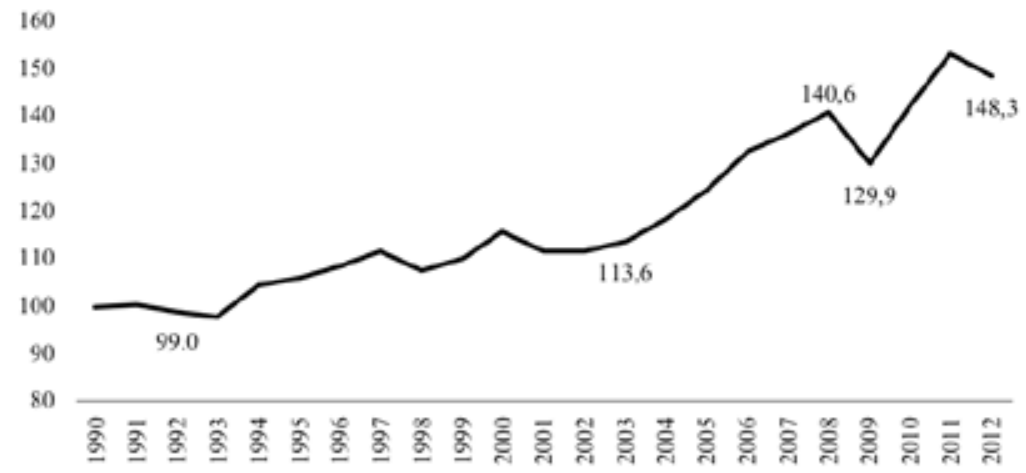

Fuente: Basado en datos de CEPAL (2013a).

Gráfico 3. América Latina: evolución del volumen de exportaciones e importaciones de bienes, 2003-2012

(indice $2003=100$ )

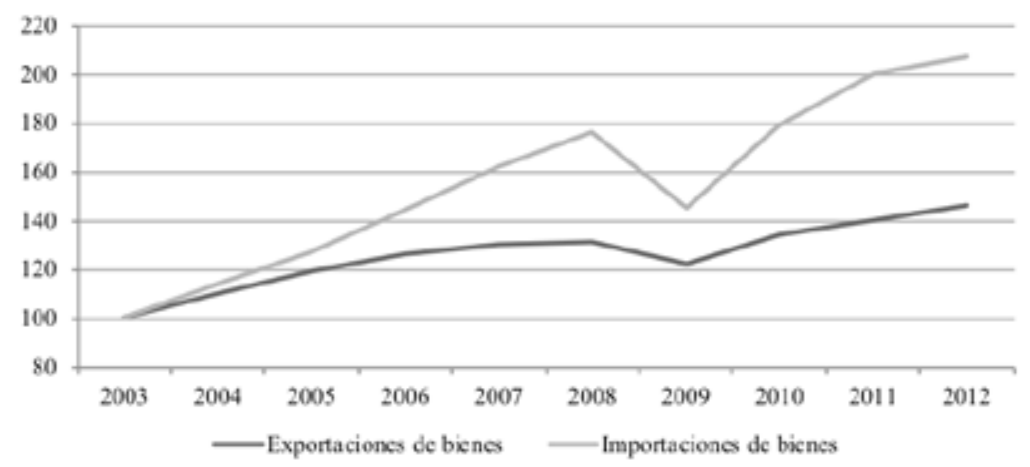

Fuente: Basado en datos fob de CEPALStat para 2003-09 y Estudio Económico de 2013 para 2010-12. Cifras provisionales para 2012.

En resumen, en paralelo a los mencionados equilibrios nominales -de baja inflación y disciplina fiscal-, se observan intensas 
fluctuaciones de la tasa de expansión del PIB, que es el principal indicador de la economía real. Mayoritariamente para el conjunto de la región, los cambios en la evolución del PIB no responden a cambios abruptos estructurales o en la microeconomía, sino que a variables macroeconómicas, predominantemente asociadas a cambios en los flujos de capitales financieros desde y hacia el exterior y a variaciones de los términos del intercambio. Ellos provocan -con su impacto multiplicado por políticas macroeconómicas pasivas o pro-cíclicas (Kaminsky, Reinhart y Vegh, 2004; Ffrench-Davis, 2010; Ocampo, 2011)- sustanciales variaciones de la demanda agregada, del tipo de cambio, y de las expectativas de los agentes económicos. Indudablemente, se trata de variables macroeconómicas, que revelan desequilibrios graves de la economía real, a pesar de que la inflación y las cuentas fiscales estén bajo control. Como se documentará a través de este artículo, esta inestabilidad ha tenido decisiva influencia negativa sobre el crecimiento del PIB y la equidad mediante su impacto en el coeficiente de inversión productiva y sobre la calidad del empleo.

La lección para el diseño de la política macroeconómica es que no basta con mantener una tasa de inflación baja y orden fiscal. Ello ha implicado un desbalance entre objetivos; naturalmente, ambos son necesarios, pero es determinante el cómo se logran en tanto que, además, por si solos resultan insuficientes para asegurar la estabilidad con crecimiento de la economía real, en circunstancias que la eficiencia exige un balance entre objetivos y la consiguiente coordinación de los medios para lograrlos. Se precisa pasar a un enfoque que prioriza, explícitamente, la interrelación de las políticas macroeconómicas con el desarrollo productivo y el impacto sobre la igualdad.

\section{ASIMETRÍAS RECESIVAS Y REGRESIVAS}

Un rasgo notorio presente en el desempeño de las economías de la región ha sido una fuerte ciclicidad de su actividad económica, con pronunciados auges seguidos por contracciones que habitualmente son abruptas; ello ha solido ser más marcado en las economías 
medianas y grandes que resultan más atractivas para los flujos financieros. Esa ciclicidad surte efectos negativos sobre la capacidad productiva y sobre su distribución entre los diversos productores del PIB (trabajadores y dueńos del capital). La capacidad productiva o PIB potencial $\left(\mathrm{PIB}^{*}\right)$ pone límite al máximo PIB efectivo obtenible en cada momento del tiempo. En consecuencia, solo un $\mathrm{PIB}^{*}$ en expansión -cuya evolución depende de los insumos y productividad que se le aporten y del entorno macroeconómico que enfrente- da sostenibilidad a un PIB efectivo creciente.

La mencionada ciclicidad ha estado asociada a grandes altibajos de la demanda agregada, del acceso al crédito, de los tipos de cambio y de los balances externos. Su variabilidad, dada la existencia de H.E., afecta de manera diferenciada o asimétrica a los diversos productores del PIB; algunos ejemplos son ilustrados en el recuadro 1.

RECUADRo 1. AsIMETRÍAS DEPRESORAS Y REGRESIVAS

- En el ciclo económico, frecuentemente, el PIB efectivo no circula alrededor del PIB potencial sino bajo él. El techo es un máximo no una media tendencial. Por ello, la inestabilidad suele no ser neutra sino ser depresora de la PTF efectiva y del empleo, y la brecha recesiva (brecha entre PIB efectivo y potencial) deprime la formación bruta de capital.

- La capacidad asignadora del tipo de cambio se resiente con la inestabilidad de la tasa libre y castiga al valor agregado de las exportaciones y la competitividad de las PYMEs frente a las importaciones.

- El inversionista financiero se puede ajustar instantáneamente frente a cambios abruptos de expectativas y de precios relativos. El inversionista productivo -el de la FBKF- cuya asignación de recursos es irreversible, requiere plazos extensos. La inestabilidad favorece la inversión especulativa versus la productiva. Esto deprime el crecimiento.

- En el ciclo se reduce la tasa de participación laboral y el empleo de los de menor empleabilidad que son los más vulnerables y se eleva la informalidad. Esto es un efecto regresivo.

- Un enfoque macroeconómico que descuida la estabilidad de la economía real resulta negativa para el crecimiento y la equidad: combina la dupla de depresora y regresiva. 
La inestabilidad de la economía real implica que, en los contextos recesivos, el PIB efectivo puede estar muy por debajo del PIB* por prolongados plazos, lo que afecta su evolución futura, por sus efectos depresivos sobre la inversión productiva, el empleo y la innovación. En cambio, en los períodos de auge, sólo por plazos breves el nivel del PIB efectivo puede superar al nivel del PIB* coetáneo. Naturalmente, durante la recuperación, el PIB efectivo suele aumentar más rápido que el PIB*, pero aún seguir permaneciendo por debajo del nivel de éste hasta alcanzar el pleno empleo. La brecha entre ambos es lo que llamamos brecha recesiva (BR), por estar subutilizando capital y trabajo disponible. El hecho que en contextos de fuerte inestabilidad la economía no fluctúe alrededor del PIB potencial, sino que principalmente bajo él, implica una asimetría notablemente relevante para el crecimiento y su distribución.

Mientras subsista la brecha entre el PIB efectivo y el potencial, persisten los efectos depresivos sobre la formación de capital, la producción de transables y su valor agregado y la calidad del mercado laboral, como se expone enseguida. Por consiguiente, es de gran relevancia, para la contribución al crecimiento y la equidad, que la actividad económica pueda permanecer en las cercanías de la frontera productiva y con ello contribuir a elevarla; la requerida cercanía no ha sido frecuente ni continuada. En la mayoría de los años entre 1981 y 2012 han prevalecido significativas BR para el conjunto de la región.

\section{A) Brecha ReCESIVA Y FORMACión de CAPITAL}

El desafío que plantea la información disponible es explicar por qué la formación bruta de capital ha sido tan reducida durante el predominio de las reformas neoliberales. Una causa principal es la existencia de desequilibrios de la economía real, consistentes en brechas significativas entre el PIB efectivo y el potencial. Como lo documentan numerosos antecedentes empíricos, la existencia de una BR entre PIB efectivo y potencial -cuya presencia constituye un desequilibrio macroeconómico fundamental- ejerce un impacto marcadamente depresivo sobre la tasa de inversión, variable 
determinante del crecimiento económico. De hecho, la experiencia latinoamericana muestra una elevada correlación negativa entre la magnitud de la brecha y la tasa de formación de capital. ${ }^{5} \mathrm{La}$ estrecha asociación que ha existido entre la BR y la FBKF en América Latina refleja uno de los principales efectos dinámicos, negativos, de la subutilización de los factores productivos.

Esta relación negativa responde a diversos factores: (i) una BR implica que se subutiliza capacidad disponible, lo que reduce la productividad efectiva (la medida standard de PTF residual); (ii) si las ventas declinan, ello indica que no se justifica expandir la capacidad hasta que el empresario prevea que se acerca a la utilización plena en su firma; (iii) las menores utilidades proveen menos fondos propios para financiar nuevas inversiones, en tanto que se desalienta la disposición a arriesgar fondos o patrimonio a largo plazo, que es lo que involucran las inversiones irreversibles; (iv) el correspondiente deterioro de los balances empresariales suele traer asociada una reticencia pro-cíclica del mercado de capitales a financiar empresas con falta de liquidez en situaciones recesivas; (v) el entorno volátil y de incertidumbre, disuade la inversión irreversible; (vi) la brecha recesiva y sus fluctuaciones suelen afectar la calidad de la evaluación de los proyectos y desalentar la innovación productiva; (vii) las intensas fluctuaciones recesivas tienden a deprimir los ingresos fiscales, induciendo recortes en la inversión pública complementaria de la privada (Easterly y Servén, 2003). ${ }^{6}$

Puede constatarse que resulta un conjunto de contundentes razones, ligadas a desequilibrios de la macroeconomía real, que explican que la insuficiente tasa de formación de capital aparezca tan asociada a los ciclos económicos. El impacto macroeconómico procíclico depresivo sobre la formación de capital opaca los esfuerzos, más estructurales, que ha estado realizando la región para elevar la productividad y reducir la heterogeneidad estructural mediante reformas micro y mesoeconómicas.

Si se evita la reaparición recurrente de la BR, con una política contra-cíclica que logre establecer una demanda agregada que se mantenga cerca del PIB potencial y con un TCR sostenible, los 
potenciales inversionistas se sentirán estimulados a intensificar su actividad. El efecto dinámico será mucho más significativo si se generan expectativas sólidas entre los actores económicos respecto de que las políticas públicas mantendrán los equilibrios de la economía real, y si, además, las autoridades emprenden reformas para completar los mercados de capitales de largo plazo, estimulan la innovación productiva y mejoran la capacitación de la fuerza laboral.

A medida que la BR va desapareciendo, muchos emprendedores que han desactivado proyectos en ciernes intentarán reactivarlos. Para ello requieren tiempo, dado el conjunto de ingredientes que suele involucrar la concreción de un proyecto de inversión productiva. En cuanto el logro de la eliminación de la brecha sea de corta duración, debido a desequilibrios que se van gestando durante la recuperación de la actividad económica, muchos potenciales inversionistas no alcanzarán a concretar su emprendimiento antes de la llegada de la próxima recesión. Es otra asimetría de los efectos cíclicos, que ayuda a explicar el deficiente desarrollo, con una menguada formación de capital, alcanzado con las políticas neo-liberales.

En este sentido, la sostenibilidad del ciclo expansivo es crucial para potenciar la inversión productiva (Titelman y Pérez Caldentey, 2015). El gráfico 4 ilustra el punto, al mostrar cómo, en los procesos de recuperación, luego de cierto rezago, la FBKF va tomando velocidad, cuya aceleración se detiene y revierte con la llegada de la nueva recesión. Por lo tanto, mientras más prolongada sea la cercanía al pleno uso, mayor tiende a ser la elevación de la tasa de inversión. En consecuencia, es imprescindible que durante el auge no se vayan gestando desequilibrios de la macroeconomía real, tales como una creciente apreciación cambiaria con un alza de la importaciones persistentemente mayor que el de las exportaciones, endeudamiento elevado de los consumidores, y/o una demanda agregada que excede en demasía la capacidad productiva. 
Gráfico 4. América Latina: formación bruta de capital, 1970-2012 (\% del PIB, dólares constantes de 2005)

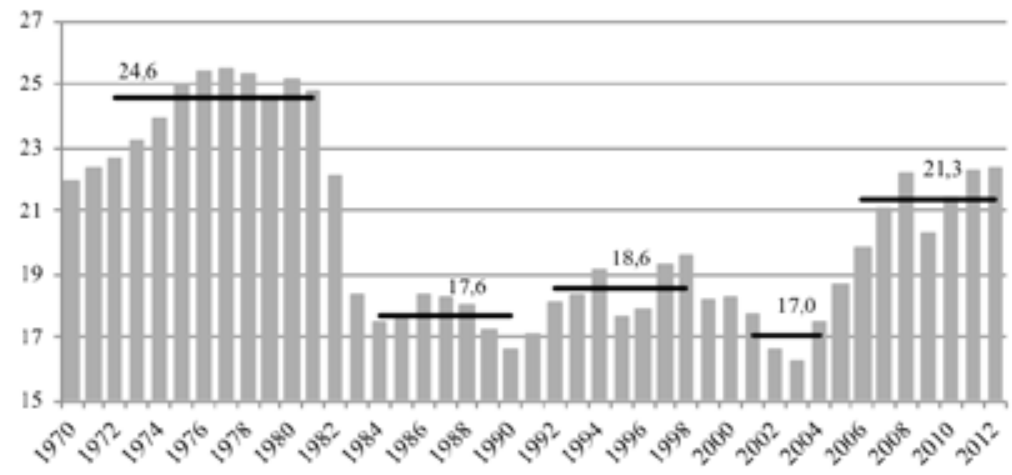

Fuente: CEPAL, cifras para 19 PALs. Las rectas horizontales indican tasas promedias en sub-períodos de FBKF/PIB en expansiones de actividad (1972-81, 1992-98 y 200612) y en contracciones recesivas (1984-90 y 2001-04).

Como lo hemos reiterado, lo común desde los 80 s ha sido que la economía inicie un proceso de reactivación, llegue a un peak que se aproxima a pleno uso de la capacidad y enseguida transite hacia una nueva recesión; en este tercio de siglo poco tiempo ha permanecido con uso elevado de su capacidad productiva. En efecto, todos los años 80s se caracterizaron por una BR significativa, luego en 1994 se alcanzó un peak con caída en 1995, otro peak surgió en el curso de 1997-98 seguido por una contracción ya en el mismo 1998, otro se inició en el curso de 2003-04 detenido por el breve contagio de 2008-09 y se retornó a un auge en 2010-12 y desaceleración en este año.

En resumen, en cada situación recesiva, la formación de capital se contrae significativamente y con los auges se suele reactivar solo gradualmente y con un rezago, denotando otra asimetría muy relevante. Por consiguiente, queda una brecha que deprime la suma de la serie de flujos de inversión durante el proceso de ajuste, aunque al final del ciclo se retorne a un flujo marginal similar al inicial. La región, desafortunadamente después de los 70s, no ha logrado sostener procesos prolongados de producción cercana al PIB 
potencial $\left(\mathrm{PIB}^{*}\right)$. Lo común ha sido que prevalezcan elevadas tasas de sub-utilización, resultante de la inestabilidad macroeconómica real, generada por flujos de capitales y precios de exportación volátiles y políticas macroeconómicas pro-cíclicas, lo que impide que la FBKF alcance a "entrar en régimen normalmente elevado".

\section{B) INeSTABILIDAD CAMBIARIA Y DESARROLlo PRODUCTIVO ${ }^{7}$}

La macroeconomía para el desarrollo, otorga un papel estratégico al tipo de cambio como el precio relativo que vincula a la economía nacional con la internacional, una variable esencial para la sostenibilidad de los equilibrios macroeconómicos y la asignación de recursos. En efecto, es una variable determinante para las decisiones de asignación de recursos productivos y de consumo de los agentes económicos, entre transables y no transables. Tanto su nivel real promedio como su estabilidad son cruciales; la existencia de heterogeneidad estructural y asimetría de respuestas implica que su inestabilidad tiende a acentuar la intensidad de la heterogeneidad.

Formalmente, varios países de la región adoptaron un régimen de tipo de cambio libre, con intervenciones esporádicas o excepcionales en algunos casos. La experiencia de la región es que el tipo de cambio real (TCR) ha exhibido un comportamiento extremadamente procíclico; ha sido liderado por el hecho que ha predominado, en la determinación de su precio de mercado, la calidad de activo financiero en vez de asignador de recursos entre transables y no transables: el TCR ha respondido con gran intensidad a variaciones de la balanza de pagos más que a la cuenta corriente. En efecto, el tipo de cambio, por prolongados períodos, ha sido determinado por flujos de capitales de corto plazo, operados por especialistas en generación de ganancias de capital, en vez de ganancias de productividad, o por variaciones reversibles de los términos de intercambio. Ello, por prolongados períodos, sin tomar en consideración si se registra una evolución desalineada de la cuenta corriente. Dado lo prolongado de los procesos revaluatorios, que implican una "volatilidad de mediano de plazo", surten efectos estructurales en la asignación de recursos; por ejemplo, desaliento de la adición de valor a las exportaciones de 
recursos primarios ${ }^{8}$ durante los períodos de apreciación cambiaria, no compensados por los efectos de las devaluaciones habituales en los períodos de explosiones recesivas.

Es efectivo que el predominio del régimen de tipo de cambio libre durante los años dos mil evitó crisis cambiarias características de un tipo de cambio nominal fijo; esto es, agotamiento de reservas internacionales y trabas arbitrarias a las importaciones, seguidas por devaluaciones masivas. Sin embargo, su contraparte ha sido que, en muchos países de la región, su cotización pasase a ser extremadamente sensible ante cambios pro-cíclicos en la oferta de fondos externos. De este modo, se incurrió en una contradicción profunda, con graves consecuencias negativas para la asignación de recursos y, en especial, para su acumulación para sustentar el crecimiento. En efecto, con las reformas liberalizadoras de las restricciones cuantitativas y arancelarias de las importaciones se procuraba un papel protagónico de los rubros transables, implicando que el tipo de cambio adquiría un papel determinante de la competitividad internacional.

Se suele argumentar que las fluctuaciones cambiarias, tan intensas, se resuelven con operaciones de derivados, compra/venta de futuros. Estos, cuando el exportador puede acceder a ellos, son muy eficaces para asegurarse a corto plazo frente a fluctuaciones de precios y tasas de interés en operaciones con la producción y las relaciones de precios actuales. Sin embargo, la realidad es que los precios de futuros son bastante similares al precio contado (spot), y tan fluctuantes uno como el otro. No son útiles para evitar los efectos distorsionadores de la inestabilidad de mediano plazo contra la formación de capital tan fundamental para el desarrollo productivo.

Es así que otra asimetría suele surgir. Durante los auges cíclicos, cuando las expectativas mejoran y los mercados de capitales se tornan más receptivos de nuevos proyectos, lo predominante ha sido que el tipo de cambio real se empiece a apreciar y a proveer la expectativa de que se trata de un proceso persistente. Ello, naturalmente, desalienta la inversión en la producción de rubros transables y en elevar su valor agregado, no obstante, lo cual a veces la apreciación 
ha sido bienvenida por las autoridades en la medida que las metas de inflación tienen preeminencia sobre el desarrollo exportador, el crecimiento y el empleo sostenible; es un ejemplo evidente de metas de inflación logradas a expensas directamente del crecimiento. En el intertanto, ha sido común que el volumen de las importaciones se eleve notoriamente más rápido que las exportaciones, y que se deteriore la cuenta corriente estructural.

En la otra etapa del ciclo, durante la contracción, suelen predominar fuertes devaluaciones cambiarias, lo que tendería a estimular la inversión en transables. Sin embargo, habitualmente, la incertidumbre es intensa y las entidades financieras tienden a restringir el financiamiento de nuevos proyectos y de PYMEs. En consecuencia, entonces, en el mercado se desaprovechan las oportunidades que ofrece un tipo de cambio depreciado para la expansión de la capacidad de producción de transables. El resultado neto, a través de ambas etapas del ciclo, es que el poder asignador positivo del tipo de cambio se debilita, y la producción de transables y su valor agregado se deprimen.

Evidentemente, la inestabilidad cambiaria distorsiona la evaluación de proyectos para la asignación de recursos, promueve la inversión especulativa por sobre la productiva, desplaza artificialmente la producción nacional de transables importables (muchos producidos por PYMEs), y desalienta la generación de valor agregado a las exportaciones.

Esta grave falla de la política cambiaria es un pesado lastre para una estrategia de desarrollo liderada por las exportaciones, especialmente las no tradicionales y con mayor valor agregado; éstas son las más propensas a transmitir externalidades y a interactuar con las PYMEs. La adopción de alguno de los diversos regímenes de flexibilidad administrada del tipo de cambio es un componente esencial del conjunto de variables requeridas para lograr éxito en esa estrategia.

Para ello es fundamental la intervención equilibradora, con un conjunto de políticas contra-cíclicas por parte de la autoridad económica; por ejemplo, mediante compra/venta de moneda 
extranjera por el Banco Central, regulación del nivel y composición de los flujos de capitales, y mecanismos de estabilización de ingresos de exportaciones. Visiones frecuentes en la literatura económica y medios de comunicación suelen afirmar que cualquier intervención cambiaria implica ir contra "el mercado", y que eso siempre resulta derrotado por "el mercado". Dado que no existe un mercado único, sino diversos mercados o segmentos, con actores o comportamientos notablemente herogeneos en su capacidad actual y su reacción, resulta esencial definir cuál mercado es el más relevante para el logro del desarrollo. Se trata de que la evolución del TCR esté acorde a sus fundamentos económicos (principalmente la tendencia de la cuenta corriente y de las productividades relativas a la Balassa/Samuelson); ello, en un mundo donde la moneda extranjera es en parte un activo financiero con una evolución frecuentemente desligada del desempeño de la cuenta corriente, hasta que los operadores de un mercado desregulado se dan cuenta de que el TCR está muy desalineado y lo realinean abruptamente.

Entonces, de lo que se trata con la intervención contra-cíclica en el mercado cambiario, es lograr que las fuerzas reales del mercado -productores de exportables e importadores y productores de importables, que son los actores más relevantes para el desarrollo productivo en lo que respecta a la relación con la economía internacional-, sean las que predominen en la determinación del tipo de cambio. Este es "el mercado" que debe imponerse, el de los generadores de mayor inversión productiva, innovación y productividad, y no el mercado de los operadores de corto plazo y buscadores de "rentas" (rent-seekers). Ello requiere que la evolución del tipo de cambio (o canasto de monedas) sea guiada por la autoridad procurando mantener una cuenta corriente sostenible.

Con ese objetivo, la autoridad puede recurrir a alguna de las variantes de flexibilidad administrada del tipo de cambio, adecuada a las particularidades de cada país (ver Williamson, 2008). Para ello es fundamental la intervención coherente y selectiva de la cuenta de capitales por parte de la autoridad económica, en combinación con un conjunto de otras políticas macroeconómicas que le provean eficacia. 
Sin esa regulación, dados los mercados financieros internacionales actuales, no hay espacio para una efectiva macroeconomía para el desarrollo, por lo tanto, con el consiguiente costo tanto en términos de crecimiento económico como de construcción de mayor igualdad en las estructuras productivas. Es una grave contradicción aspirar a una eficiente economía de mercado, en una economía en desarrollo, y que las autoridades deleguen en operadores financieros la determinación de precios macroeconómicos tan estratégicos como lo es el tipo de cambio.

En síntesis, para ser consistentes con una estrategia de desarrollo en la que la producción de bienes y servicios transables internacionalmente tenga un papel central, se requiere una profunda corrección de la política cambiaria, para así fortalecer su calidad como asignador de recursos productivos y para intensificar la vinculación (o encadenamientos de la producción de transables) con el resto de la economía. Esta vinculación mayor con los segmentos de la economía que no exportan directamente puede contribuir a reducir la heterogeneidad estructural interna, lo que generaría condiciones más igualitarias en lo laboral y entre la gama de empresarios de diversos tamańos.

C) EMPleos de CALIDAd E INESTABILIDAd DE LA ECONOMÍA REAL ${ }^{10}$ La situación laboral, en particular en economías con cargas tributarias bajas y modestos niveles de gasto social -como es, en general, el caso de América Latina- es la variable más determinante de la distribución del ingreso.

A pesar de los progresos en reducción de la pobreza desde los años noventa, según la definición habitual (CEPAL, 2013b), la situación laboral se encuentra aun sin lograr un avance sostenido hacia un predominio de empleos más estables, con protección social, organización y capacidad de negociación de los trabajadores. No obstante, los progresos significativos registrados durante la recuperación económica y social de 2004-08, la precariedad laboral aun predominaba antes del contagio de la crisis global financiera; naturalmente, las falencias se agravaron con el contagio. La pobreza 
y la precariedad laboral se deterioraron cuando el auge se detuvo bruscamente en el 2009. En lugar de seguir dando paso a empleos más formales, la informalidad recuperó espacio. Entre 2007 y 200910 la participación en el empleo de los cuenta propia, "involuntarios" en su mayoría, se incrementó (OIT, 2010). La positiva recuperación desde 2010 retomó la senda de progreso de 2004-08. Ello permitió elevar el empleo, la formalidad y los ingresos, y el PIB efectivo hasta topar con la limitada capacidad productiva disponible entre 2011 y 2012, y enfrentar el desafío de elevar la generación de PIB potencial, dado que solo había crecido levemente.

La generación de más y mejor empleo es determinante para el logro de una progresiva reducción de las intensas desigualdades que se observan en los mercados y sociedades. En consecuencia, la política macroeconómica debiera prestar atención explícita a los efectos diferenciados, de diversas políticas, sobre empresas grandes y pequeñas, sobre inversión y consumo, sobre trabajadores calificados y de baja calificación. La gradualidad de las políticas y la calidad de la coordinación entre los aspectos monetarios, cambiarios, cuenta de capitales, financieros y fiscales, por ejemplo, hacen una diferencia sustancial para el crecimiento económico y sus efectos distributivos, en particular sobre el nivel y calidad del empleo.

Subsiste una notoria brecha social, pues la razón entre quintiles de ingresos más rico y más pobre (Q5/Q1) de los PALs que duplica la del G-7. En efecto, la región sigue siendo muy regresiva en el contexto mundial (Banco Mundial, 2005) y ello está asociado, de manera determinante, a las estructuras productivas. Cabe reiterarlo, la fuerte heterogeneidad estructural entre empresas de diferentes dimensiones y entre trabajadores de diversa calificación, implica desigualdades generadas en el correspondiente funcionamiento de los mercados. ${ }^{11}$ Para crecer vigorosamente se precisa mejorar más rápidamente la productividad de los sectores de menores ingresos, y con ello la empleabilidad de los sectores medios y pobres. En contraposición a la creencia más común en el enfoque neoliberal, en la situación actual de América Latina hay un amplio campo de complementariedad entre políticas que contribuyan 
simultáneamente al crecimiento y a la reducción de la desigualdad en el mercado laboral (ver Bourguignon y Walton, 2007; FfrenchDavis, 2014, cap. VII).

La inestabilidad de la demanda interna y del tipo de cambio involucra efectos estáticos y dinámicos sobre el empleo. Estáticos, tales como altibajos de la tasa de utilización de la capacidad productiva disponible de trabajo y capital, que al provocar, recurrentemente, brechas sustantivas entre la capacidad instalada y el PIB efectivo, generan también brechas entre el pleno empleo y el empleo efectivo. Esas BR, y la volatilidad de variables como el tipo de cambio real, han implicado profundos efectos dinámicos, como se expuso recién, (i) sobre la expansión del stock de capital, la que se restringe en tanto que la población en edad de trabajar sigue elevándose; (ii) se debilitan las organizaciones laborales y se intensifica la discriminación contra los trabajadores de menor calificación en situaciones de creciente desempleo y contra los empresarios de menor tamaño; (iii) se desalienta la intensidad del valor agregado incorporado en las exportaciones y su interrelación con el resto de la producción interna; (iv) sobre el desarrollo de las PYMEs que suelen ser más intensivas en el trabajo, y que compiten con las importaciones, y (v) sobre la formalidad o precariedad del empleo y la tasa de participación laboral.

La aparición de una BR provocada por la inestabilidad macroeconómica suele venir acompańada por un aumento de los trabajadores en la informalidad y por una menor tasa de participación laboral. Ambos implican que la información sobre la tasa global de desempleo subestima el deterioro de la situación laboral durante los ajustes recesivos. En efecto, los empleos asalariados, con contratos y seguridad social pierden peso relativo a favor de la informalidad y la tendencia al aumento de la participación laboral se debilita ante el hecho de que hay personas en edad de trabajar que dejan de buscar trabajo en las situaciones de brecha recesiva (FfrenchDavis, 2012, pp. 28-31); estos no aparecen entre los desocupados, lo que implica subestimar la magnitud del desequilibrio laboral que debería enfrentarse. 
Un vínculo entre inestabilidad de la macroeconomía real y la desigualdad reside en la profunda heterogeneidad estructural que caracteriza a las economías en desarrollo. Lo reiteramos, dada la fuerza que aún tiene el enfoque neoliberal y su creencia en la homogeneidad y "neutralidad" de políticas, es fundamental comprender e internalizar en el diseño de políticas públicas la diversa capacidad de acción y reacción de los agentes típicos en distintos mercados. Como ser grandes y pequeńos empresarios; trabajadores de elevada y de reducida calificación; inversionistas productivos e inversionistas financieros o compradores de activos existentes; inversionistas productivos y consumidores; alta movilidad nacional e internacional de los capitales financieros y del trabajo muy calificado, en contraste con la escasa movilidad del capital físico y de la mano de obra de menor calificación.

Las asimetrías resultantes de esa heterogeneidad se intensifican ante la inestabilidad de la actividad económica y los macro-precios. Por ejemplo, la distribución del ingreso tiende a deteriorarse en las recesiones y a mejorar en las recuperaciones, pero más gradualmente en los auges y más abruptamente en las contracciones; esta asimetría deja balances con efectos regresivos en los hogares y las firmas. Adicionalmente, tienden a debilitarse los esfuerzos en aquellas reformas económicas que requieren continuidad y una perspectiva de largo plazo. A su vez, durante los auges de flujos financieros, parte sustancial de ellos se ha consumido, debido a que el consumo responde con mayor rapidez que la inversión productiva ante la mayor oferta de fondos y el mercado financiero se ha especializado en aquel destino más que en el financiamiento de largo plazo de la inversión productiva. Si se acompańa, como ha sido frecuente, por apreciaciones cambiarias, se acentúa el sesgo con crecientes importaciones de bienes de consumo lo que desvía el "ahorro externo" hacia "desahorro" nacional.

Entonces, después del auge quedan elevados pasivos sin una contrapartida de capacidad de pago; el habitual cambio posterior de expectativas, reversión de flujos y devaluaciones abruptas, conducen a un ajuste recesivo, con caídas de la demanda interna. A su vez, ésta arrastra a la baja a la producción, el empleo y su formalidad 
y los ingresos tributarios. A ello se ha estado dirigiendo la región en los años recientes con la aun reducida tasa de FBKF a pesar de notables precios de exportación e ingreso de capitales extranjeros y un creciente desequilibrio entre volúmenes de exportaciones e importaciones (ver Recuadro 2 y gráfico 3). Cabe agregar, la débil institucionalidad de la protección social para transferir ingresos en casos de crisis, que posean un signo contra-cíclico y progresivo, ya sea para reinserción, capacitación o compensación de pérdidas de ingresos (OIT/CEPAL, 2011).

\section{RECUADRO 2. LA COYUNTURA LATINOAMERICANA EN 2012}

- Período coyuntural positivo para América Latina en 2010-12, luego de uno similar en 2004-08. Se posiciona en el techo o PIB potencial en 2012 lo que explica el freno del aumento del PIB efectivo ya en este año.

- Shocks de términos del intercambio y financieros positivos elevaron la demanda interna en 2004-08 y 2010.12, en un entorno inicial con una restricción externa dominante en 2003 y 2009. Por ello, pudieron tener un fuerte efecto reactivador del PIB, arrastrando a la economía real hasta el techo (positivo) y apreciando el tipo de cambio (negativo). No hay cambio estructural o en la política macroeconómica radical que expliquen los dos saltos en el crecimiento del PIB efectivo; sí lo hacen los shocks externos positivos por su impacto en la demanda agregada y con ello en la tasa de utilización de la capacidad disponible.

- Las exportaciones siguen muy concentradas en recursos naturales, con bajo valor agregado y precios inestables cíclicamente. En este período de altos precios, el volumen de las importaciones creció notablemente más rápido que él de las exportaciones.

- La formación bruta de capital se recuperó y en los años más recientes alcanzó el mayor nivel desde la implantación del enfoque neoliberal del Consenso de Washington. Ese logro es, sin duda, muy positivo, y es explicado principalmente por el cierre de la brecha recesiva entre PIB efectivo y potencial por un lapso más prolongado que en los anteriores ciclos (alrededor de 8 años, ver gráfico 4). Sin embargo, la tasa de inversión productiva aún es inferior al promedio del decenio previo a la crisis de la deuda, y el ahorro nacional (controlando debidamente por el efecto de los términos del intercambio) se ha debilitado. La región está dependiendo, riesgosamente, de términos del intercambio comercial y flujos financieros transitoriamente muy favorables. 
Como se expuso, la evolución tan cíclica del TCR de muchos países ha distorsionado las decisiones de inversión productiva. Es efectivo que durante los auges acompańados de apreciación cambiaria, se suelen generar excesos en la construcción y en la comercialización de importaciones que proveen empleos que no serán sostenibles cuando se corrija un déficit externo que deviene crecientemente excesivo. En contraparte, desplazan artificialmente producción de transables importables que compiten con las importaciones (muchos producidos por PYMEs, como ya se señaló); al mismo tiempo, desalientan la diversificación hacia rubros no tradicionales más intensos en valor agregado y la adición de valor a las exportaciones tradicionales, con efectos negativos sobre el empleo sostenible y su calidad. En los auges, por lo tanto, parte de la creación de empleos es transitoria, mientras que se provoca cierta destrucción de empleos permanentes.

La inestabilidad de la economía real es asimétrica en lo distributivo e inevitablemente implica subutilización de la productividad potencial, con una menor producción efectiva, y menor empleo con expansión de los sectores informales en comparación con contextos de mayor estabilidad de la economía real. Resulta evidente que tasas más altas de utilización del capital suelen implicar que el nivel promedio del empleo es superior y que la fuerza laboral se combina con un stock más elevado de capital físico en uso. El consiguiente aumento de la productividad observada significa que el bienestar de los trabajadores y los inversionistas (salarios y utilidades del capital) pueda mejorar en virtud de la mayor utilización de la capacidad e incentivar un círculo virtuoso de mayor inversión, innovación y empleos. Es la tarea de las políticas macroeconómicas, que deben ser complementadas por políticas de desarrollo productivo, capacitación y reforma incluyente del mercado de capitales.

En resumen, los profundos ciclos que sufre la actividad económica, por inestabilidad de la demanda agregada y del tipo de cambio, naturalmente afectan el nivel de empleo, su formalidad, la naturaleza de los contratos laborales, y la evolución de los salarios. La inestabilidad de la macroeconomía real, dada la heterogeneidad 
estructural vigente en nuestros mercados, arroja un impacto netamente regresivo sobre la distribución del ingreso y la calidad de los empleos.

\section{Conclusiones}

El entorno macroeconómico surte diversos efectos sobre el desarrollo. Entre ellos se encuentran su impacto (i) sobre la tasa de inversión productiva, (ii) sobre la intensidad del valor agregado generado en las exportaciones y su interrelación con el resto del PIB, (iii) sobre la innovación y su distribución entre diferentes sectores de la economía, (iv) sobre el desarrollo de las pequeñas y medianas empresas; (v) sobre la formalidad o precariedad del mercado laboral, y (vi) sobre el presupuesto fiscal. Como se expuso, el estilo de hacer políticas macroeconómicas tiene una significativa incidencia en todas estas variables.

Los inversionistas productivos y el empleo han estado sujetos a gran inestabilidad de la macroeconomía real, con elevadas brechas recesivas, en un mercado financiero notoriamente incompleto, en particular en el caso del financiamiento de las empresas de menor tamaño. A su vez, la inestabilidad de la economía real ha estado asociada, estrechamente, a ciclos de los flujos financieros con el exterior, los que no solo exhiben alta volatilidad pro-cíclica sino también una escasa conexión con la inversión productiva; ha predominado lo que llamamos financierismo versus el productivismo (Ffrench-Davis, 2010). La causa central es un mercado financiero internacional dominado por agentes especializados en el corto plazo, cuyo comportamiento suele ser intrínsecamente pro-cíclico y circular principalmente al margen del financiamiento de la inversión productiva. Esa deficiencia es agravada por el hecho que su volatilidad ha dado lugar a crisis financieras y cambiarias en las economías emergentes receptoras de esos flujos, cuyos efectos recesivos han desalentado la formación de capital y el empleo. En efecto, la creciente integración con los mercados financieros internacionales más volátiles -que suele implicar la apertura indiscriminada de la cuenta de capitales-, ha incidido en una 
profundización de la inestabilidad, con una superficialización del financiamiento. No puede ignorarse, un hecho tan evidente, que es el gran incremento del 'ahorro financiero' y la persistencia de tasas de inversión productiva aún bajas y concentradas.

Como las fluctuaciones no son simétricas alrededor del pleno empleo, sino que asimétricas, con un definido sesgo depresivo y regresivo, habitualmente las economías de la región se han encontrado con niveles promedios de actividad sustancialmente por debajo del pleno empleo; esto es, con significativas brechas recesivas. Ello involucra un decisivo desaliento para la inversión productiva y un sesgo regresivo o de inequidad por su impacto negativo sobre la calidad del empleo y contra las empresas de menor tamaño. En la medida que se logre estabilidad de la economía real, y así se reduzcan las brechas recesivas, ello suele contribuir a elevar la formación de capital, el empleo sostenible y el PIB potencial. Estos efectos no son neutros distributivamente, dada la heterogeneidad estructural prevaleciente, pues la existencia de la brecha suele implicar impactos negativos particularmente fuertes para las PYMEs, los trabajadores de menor calificación y los sectores con menor patrimonio. La heterogeneidad en el acceso al financiamiento refuerza las desigualdades en capacidades productivas, en un círculo vicioso que condena a las unidades productivas de menor capital a la vulnerabilidad y a la dificultad para crecer, con los consiguientes aumentos de las brechas de ingresos a causa de la inestabilidad de la macroeconomía real: el sesgo recesivo es también regresivo. Por ello, se trata de diseñar políticas que permitan potenciar y prolongar el ciclo expansivo, evitando las vulnerabilidades que han solido conducir, en cada auge, hacia nuevos ajustes recesivos; para el desarrollo productivo, resulta imprescindible la estabilidad sostenible de la demanda interna y de macro-precios como el tipo de cambio.

Adicionalmente, una reforma de las reformas neoliberales debe priorizar la vinculación del sistema financiero -tanto del mercado financiero nacional como de la cuenta de capitales- al proceso de inversión interna más que a los mercados financieros externos de corto plazo y especulativos. 
El desafío para una macroeconomía para el desarrollo es diseñar un conjunto de herramientas, identificando su dosificación principalmente de las políticas fiscal, monetaria, cambiaria, del sistema financiero nacional y la cuenta de capitales- que reconozca la interrelación entre el corto y largo plazo, que concilie la estabilidad de la economía real con un mayor dinamismo en el crecimiento de largo plazo, y que contribuya a la inclusión social. Ello requiere un conjunto de políticas, ineludiblemente armonizadas entre sí. No hay lugar para autonomías contradictorias con la coordinación, ni para el predominio de un objetivo a expensas de otros. Es preciso establecer (i) una política fiscal y tributaria consistente con el financiamiento de la agenda de desarrollo social, la inversión pública y las políticas de desarrollo productivo, (ii) una demanda agregada en niveles consistentes con el PIB de pleno empleo y (iii) una evolución del tipo de cambio sostenible en el tiempo, consistente con el balance de la cuenta corriente. Estas son condiciones básicas de los equilibrios macroeconómicos de la economía real.

En el contexto internacional actual y previsible, para su viabilidad se requiere la regulación contra-cíclica de la cuenta de capitales y mecanismos estabilizadores de los ingresos de exportaciones con precios altamente cíclicos. La regulación contra-cíclica de los ingresos y egresos de la cuenta de capitales procura lograr equilibrios sostenibles de la macroeconomía real; esto es, lo opuesto a pretender perpetuar desequilibrios. ${ }^{12}$

En efecto, en economías emergentes como las de los países latinoamericanos, una regulación contra-cíclica de la cuenta de capitales, efectiva y eficiente, provee espacio para políticas monetaria y cambiaria activas -simultáneamente contra-cíclicasy para una reorganización del sistema financiero local que apunte a canalizar recursos hacia la inversión productiva, con un sesgo inclusivo, contribuyendo a reducir la heterogeneidad estructural entre diferentes sectores productivos y sociales. Por consiguiente, emerge como una condición imprescindible para avanzar hacia una macroeconomía para el desarrollo (Ocampo, 2011).

Para crecer vigorosamente se precisa mejorar la productividad 
de los sectores medios y pobres, pues la desigualdad y la pobreza constituyen un lastre para el desarrollo económico (Bourguignon y Walton, 2005). Por ello, ocupa un lugar estratégico el diseño de un sistema financiero que contribuya a reducir la gran heterogeneidad estructural de las economías de la región. Esto implica desarrollar con fuerza el financiamiento de las PYMEs, en diferentes escalas e institucionalidades. La incompletitud de los actuales mercados de capitales ha sido una barrera infranqueable para muchas PYMEs para dar un salto en su desarrollo.

La manera cómo se aborde la reforma de los mercados de capitales nacionales y la conexión con los mercados financieros internacionales representa un desafío crucial para lograr, sostenidamente, una macroeconomía para el desarrollo, y para la disminución de la heterogeneidad estructural y de la desigualdad que ella implica. Se precisa que se vayan generando estructuras productivas que progresivamente -ineludiblemente con avances graduales pero necesariamente persistentes- involucren mercados más equitativos, gracias a que, crecientemente, se van reduciendo las brechas de productividad y de ingresos entre empresas de diversos tamaños y trabajadores de diferente origen social y capacitación, gracias a la progresiva disminución de su heterogeneidad estructural.

\section{REFERENCIAS}

AGOSIN, M. R. (2007), "Trade and growth: Why Asia grows faster than in Latin America", en R. Ffrench-Davis y J.L. Machinea (eds.), Economic Growth with Equity: Challenges for Latin America (2007), Palgrave Macmillan, Nueva York.

BANCO MUNDIAL (2005), World Development Report 2006: Equity and Development, Oxford University Press, Nueva York. BIELSCHOWSKY, R. (1998), Cincuenta años de pensamiento de la CEPAL, Fondo de Cultura Económica, Santiago.

BLANCHARD, O., G. Dell'Ariccia y P. Mauro (2010), "Rethinking Macroeconomic Policy", IMF Staff Position Note, SPN/10/03, febrero. 
BOURGUIGNON, F. y M. Walton (2007), "Is greater equity necessary for higher long-term growth in Latin America?", en R. Ffrench-Davis y J.L. Machinea (eds.), Economic Growth with Equity; Challenges for Latin America, Palgrave Macmillan, Nueva York.

CEPAL (2010), La hora de la igualdad: brechas por cerrar, caminos por abrir, Naciones Unidas, Santiago.

(2013a), Estudio Económico de América Latina y el Caribe 2013, Naciones Unidas, Santiago.

(2013b), Panorama social de América Latina, Naciones Unidas, Santiago.

(2012), Cambio estructural para la igualdad: una visión integrada del desarrollo, Naciones Unidas, Santiago.

(1998), Politicas para mejorar la inserción externa, Fondo de Cultura Económica/CEPAL, Santiago.

EASTERLY, W. y L. Servén (2003), The Limits of Stabilization: Infrastructure, Public Deficits and Growth in Latin America, Stanford University Press, Stanford, California.

EICHENGREEN, B. (2008), "The real exchange rate and economic growth", Working Paper No. 4, Commission on Growth and Development, Washington, DC.

ERTEN, B. y J.A. Ocampo (2012), "Supercycles of commodity prices since the mid-nineteenth century", World Development, vol. 44.

FFRENCH-DAVIS, R. (2014), Chile entre el neoliberalismo $y$ el crecimiento con equidad, JCSáez Editor, quinta edición, Santiago.

(2012), "Empleos de calidad y estabilidad macroeconómica real: el rol regresivo de los flujos financieros", Revista Internacional del Trabajo, Vol. 131, No.1-2, junio, OIT, Ginebra.

(2010), "Macroeconomía para el desarrollo: desde el financierismo al productivismo", Revista CEPAL No 102, diciembre.

(2005), Reformas para América Latina: Después del 
fundamentalismo neoliberal, Siglo XXI Editores/CEPAL, Buenos Aires.

(1988), "Esbozo de un planteamiento neo-estructuralista", en Revista de la CEPAL No 34, Santiago, abril.

GALLAGHER, K., Griffith-Jones, S. y J.A. Ocampo (2012), eds., Regulating global capital flows for long-run development, Boston University.

HOFMAN, A. y H. Tapia (2003), "Potential output in Latin America: a standard approach for the 1950-2000 period", Estudios estadísticos y prospectivos, No. 25 (LC/L.2042-P), Santiago.

INFANTE, R. (2011) (coord.), Desarrollo inclusivo en América Latina y el Caribe. Ensayos sobre politicas de convergencia productiva para la igualdad, Libros de la CEPAL, No112, Santiago.

KAMINSKY, G., C. Reinhart y C. Vegh (2004), "When it Rains, it Pours: Procyclical Capital Flows and Macroeconomic Policies", Working Paper 10780, NBER, enero.

KRUGMAN, P. (2011), "How did Economists get it so Wrong?", New York Times, 6 de septiembre.

MAGUD, R. y C. Reinhart (2007), "Capital controls: an evaluation", en S. Edwards (ed.), Capital controls and capital flows in emerging economies: policies, practices and consequences, University of Chicago Press, Chicago.

OCAMPO, J. A. (2011), "Macroeconomía para el desarrollo: políticas anticíclicas y transformación productiva", Revista CEPAL No 104, agosto.

OCAMPO, J. A. y J. Ros (2011), (eds.), The Oxford Handbook of Latin American Economics, Oxford University Press, Nueva York.

OCDE (2013), OECD Economic Surveys: CHILE, París.

OIT (2010), Panorama Laboral 2010, América Latina y el Caribe, Lima.

OIT/CEPAL (2011), “Coyuntura laboral en América Latina y el 
Caribe”, Boletín No 5, junio.

OSTRY, J., Ghosh, A., et al. (2011), "Managing capital inflows:

What tools to use?", IMF Staff Discussion Note, 11-06.

PREBISCH, R. (1970), Transformación y desarrollo: La gran tarea de la América Latina, Fondo de Cultura Económica, México DF. RAJAN, R. (2011), Fault lines: how hidden fractures still threaten the world economy, Princeton University Press, Princeton.

RODRIK, D. (2011), The globalization paradox, W.W. Norton, Nueva York.

(2008), "The real exchange rate and economic growth", Brookings Papers on Economic Activity, Washington, D.C., Brookings Institution Press.

SUNKEL, O. (2011), El presente como historia, Catalonia, Santiago. (1991), ed., El desarrollo desde dentro: un enfoque neoestructuralista para la América Latina, Fondo de Cultura Económica, México DF.

TAPIA, H. Y J.C. GUATAQUI (2013), “Políticas económicas para el crecimiento", borrador de trabajo, División de Desarrollo, CEPAL, Santiago.

TITELMAN, D. y E. Pérez Caldentey (2015), “Macroeconomía para el desarrollo en América Latina y el Caribe: nuevas consideraciones sobre políticas anticíclicas”, en este volumen. WILLIAMSON, J. (2008), "Do development considerations matter for exchange rate policy?", en K. Cowan y S. Edwards (eds.), Current account and external financing, Banco Central de Chile.

(2003), "Overview: An Agenda for Restarting Growth and Reform”, en Kuczynski y Williamson (2003)

Notas de PÁgina

${ }^{1}$ Publicado originalmente en CEPAL, Neoestructuralismo y corrientes heterodoxas en el siglo XXI, Santiago, 2015. Este nuevo manuscrito contiene ajustes menores. Agradezco la colaboración de Felipe Correa y los comentarios de editores de este libro y de participantes 
en el Seminario organizado por CEPAL sobre Neoestructuralismo y Economía Heterodoxa, el 22-23 de abril de 2013. Los primeros planteamientos que publiqué sobre neo-estructuralismo se encuentran en Ffrench-Davis (1988), construidos sobre los pioneros trabajos de grandes autores como Aníbal Pinto, Raúl Prebisch, Osvaldo Sunkel y Fernando Faynzylber.

${ }^{2}$ El quinquenio 2004-08 fue el más positivo desde los años 70s, con un crecimiento promedio de 5,3\% anual. En 2009-12, la mejora relativa de la región descansa más en el retroceso de las economías más desarrolladas.

${ }^{3}$ Ver CEPAL (2010) y Ffrench-Davis (2010).

${ }^{4} \mathrm{El}$ aumento de cerca de $50 \%$ en el índice de términos del intercambio sobrestima el impacto positivo sobre ingresos por exportación, pues parte del efecto se filtra al exterior a través de las remesas de utilidades de la IED instalada en el sector exportador. La filtración fue de alrededor de un tercio en el período reciente de auge. Ver Erten y Ocampo (2012) sobre tendencia de los términos del intercambio.

${ }^{5}$ En Ffrench-Davis (2005, cap. II) se examina el tema en detalle. Estimaciones para 1970-2003 están basadas en antecedentes de CEPAL y Hofman y Tapia (2003), para nueve PALs que comprenden una gran mayoría de la población y del PIB regional. El tema es retomado en Ffrench-Davis (2010), donde esas estimaciones, actualizadas por los mismos autores, cubren hasta 2009. Las cifras de inversión se refieren a la formación bruta de capital fijo.

${ }^{6} \mathrm{El}$ impacto depresivo del ciclo sobre la FBKF es intensificado por el hecho que la contracción de la inversión suele ser más bien abrupta en contraste con una recuperación gradual. Esta nueva asimetría arroja una suma de flujos de inversión menor que la resultante si ambos ajustes fuesen simétricos.

7 Ver, por ejemplo, Williamson (2008); Agosin (2007); Rodrik (2008); Eichengreen (2008); Ffrench-Davis (2010); Ocampo (2011).

${ }^{8}$ La literatura ortodoxa es abrumadora en cuanto a sostener que el tipo de cambio plenamente flexible, libre de intervenciones por 
parte de la autoridad, es eficaz en enfrentar los ajustes de corto plazo ante los períodos contractivos, ignorando la distorsión de los efectos asignadores resultantes en el auge y en la contracción. Ver, por ejemplo, OCDE (2013, pp. 8 y 15-16).

${ }^{9}$ Como lo hemos reiterado, una reducción más significativa de la H.E. requiere la presencia simultánea de reformas estructurales y microeconómicas junto a las reformas de la macroeconomía. Cabe destacar que, a su vez, un entorno macroeconómico pro-cíclico, como los que ha generado el enfoque neoliberal, suele obstaculizar cualquiera política de desarrollo productivo incluyente. De hecho, suele debilitar la acción pública y afectar más negativamente a los sectores privados de menor productividad.

${ }^{10}$ El impacto del entorno macroeconómico y la cuenta de capitales sobre el nivel y la calidad del empleo se examina en FfrenchDavis (2012).

${ }^{11}$ Ver en Infante (2011) análisis y antecedentes empíricos sobre el intenso grado de heterogeneidad estructural actual en las economías de América Latina y sus implicancias distributivas. Ver Sunkel (2011, cap. VII) sobre la situación de Chile.

${ }^{12}$ Hay experiencias de control pro-cíclicos o que intentan perpetuar desequilibrios insostenibles. En contraste, varios países de América Latina han efectuado interesantes esfuerzos de regulación contra-cíclica (ver, por ejemplo, CEPAL, 2010, recuadro II.2). Entre ellos destaca la exitosa experiencia de Chile en el primer quinquenio de los noventa, que ilustra la eficacia que pueden alcanzar, para el desarrollo, las regulaciones contra-cíclicas de la cuenta de capitales cuando son coherentes e integrales (ver Ffrench-Davis, 2014, cap. VIII; Magud y Reinhart, 2007).

Fecha de Recepción del Artículo: 28 de abril de 2018 Fecha de Aceptación: 29 de abril de 2018 